JOURNAL OF

SYMPLECTIC GEOMETRY

Volume 7, Number 3, 295-310, 2009

\title{
CLASSIFICATION OF MULTIPLICITY FREE HAMILTONIAN ACTIONS OF ALGEBRAIC TORI ON STEIN MANIFOLDS
}

\author{
IVAN LOSEV
}

\begin{abstract}
A Hamiltonian action of an algebraic torus on a symplectic complex manifold is said to be multiplicity free if a general orbit is a lagrangian submanifold. To any multiplicity free Hamiltonian action of an algebraic torus $T \cong\left(\mathbb{C}^{\times}\right)^{n}$ on a Stein manifold $X$ we assign a certain 5 -tuple consisting of a Stein manifold $Y$, an étale map $Y \rightarrow \mathfrak{t}^{*}$, a set of divisors on $Y$ and elements of $H^{2}(Y, \mathbb{Z})^{\oplus n}, H^{2}(Y, \mathbb{C})$. We show that $X$ is uniquely determined by this invariants. Furthermore, we describe all 5-tuples arising in this way.
\end{abstract}

\section{Contents}

1. Introduction 295

2. Generalities on MF Hamiltonian Stein T-manifolds 298

3. The sheaf $\mathcal{A} u t \quad 303$

4. Uniqueness $\quad 305$

5. Existence 307

6. An open problem 308

$\begin{array}{ll}\text { References } & 309\end{array}$

\section{Introduction}

Let $X$ be a smooth manifold with a symplectic form $\omega$ and $T$ be a compact torus acting on $X$ by symplectomorphisms. We recall that the action $T: X$ is called Hamiltonian if there are $n=\operatorname{dim} T$ functions $H_{1}, \ldots, H_{n} \in C^{\infty}(X)$ such that

(H1) $\left\{H_{i}, H_{j}\right\}=0$, where $\{\cdot, \cdot\}$ denotes the Poisson bracket on $X$ induced by $\omega$. 
(H2) The skew-gradient of $H_{i}$ coincides with the velocity vector field of $\xi_{i}$, where $\xi_{1}, \ldots, \xi_{n}$ is a basis of the Lie algebra $\mathfrak{t}$ of $T$.

It follows from (H1), (H2) that all functions $H_{i}$ are $T$-invariant.

To a Hamiltonian action $T: X$ one assigns a map $\mu: X \rightarrow \mathfrak{t}^{*},\left\langle\mu(x), \xi_{i}\right\rangle=$ $H_{i}(x)$, called the moment map. This map is $T$-equivariant and satisfies the following identity:

$$
\left\langle d_{x} \mu(v), \xi\right\rangle=\omega\left(\xi_{*} x, v\right), \forall x \in X, v \in T_{x} X, \xi \in \mathfrak{t},
$$

where $\xi_{*} x$ denotes the velocity vector in $x$ induced by $\xi$. Note that the moment map is defined uniquely up to the addition of a scalar. The manifold $X$ (or, more precisely, the quadruple $(X, \omega, T: X, \mu)$ ) is called a Hamiltonian T-manifold.

We remark that, by (H1), (H2), any $T$-orbit in $X$ is an isotropic submanifold. When a general orbit is lagrangian, the Hamiltonian $T$-manifold $X$ is called multiplicity free or, shortly, $M F$ (one also uses the term symplectic toric manifold).

The most crucial result concerning compact MF Hamiltonian $T$-manifolds is their classification due to Delzant [D]. If $T_{0}$ is the inefficiency kernel of the action $T: X$ (that is, the set of all elements of $T$ acting trivially on $X)$, then the action $T / T_{0}: X$ is Hamiltonian with moment map $p \circ \mu$. Here $\mu$ is the moment map for the action $T: X$ and $p$ is the natural projection $\mathfrak{t}^{*} \rightarrow\left(\mathfrak{t} / \mathfrak{t}_{0}\right)^{*}$. So it is enough to deal with effective actions. By the famous result of Atyah $[\mathbf{A}]$, and Guillemin-Sternberg $[\mathbf{G S}]$ the image $\mu(X)$ is a convex polytope in $\mathfrak{t}^{*}$ (provided $X$ is compact). This image is called the moment polytope of $X$. Delzant proved that a compact MF Hamiltonian $T$-manifold is uniquely determined by its moment polytope. He also found a necessary and sufficient condition for a convex polytope in $t^{*}$ to be a moment polytope of a compact MF Hamiltonian manifold.

The goal of the present paper is to solve a similar classificational problem for algebraic tori actions on complex symplectic manifolds (with holomorphic symplectic form). The definition of a (MF) Hamiltonian action is generalized to the complex case directly (the functions $H_{i}$ are now taken from the algebra $\mathcal{O}(X)$ of holomorphic functions). Let $T$ denote an algebraic torus $\left(\mathbb{C}^{\times}\right)^{n}$ and $X$ a complex Hamiltonian $T$-manifold. Since the moment map $\mu$ is holomorphic, the manifold $X$ is not compact unless the action $T: X$ is trivial. In this paper we concentrate on the case when $X$ is a Stein manifold.

The data we use to classify MF Hamiltonian Stein $T$-manifolds are more complicated than in the Delzant case. Below $X$ is a MF Stein Hamiltonian $T$-manifold.

By the results of $[\mathbf{S}]$, there is a categorical quotient $Y$ for the action $T: X$, which is a Stein space (see Section 2 for details). Let $\pi: X \rightarrow$ $Y$ be the quotient map. It turns out that in our case $Y$ is even a Stein manifold (Lemma 2.7). Let $\psi$ be a unique holomorphic map $Y \rightarrow \mathfrak{t}^{*}$ such 
that $\mu=\psi \circ \pi$. One can show that $\psi$ is étale (=a local isomorphism), Lemma 2.7. The manifold $Y$ and the map $\psi$ are the first two pieces of data we need.

For a one-dimensional subgroup $T_{0} \subset T$ we denote by $\widetilde{\mathcal{D}}\left(T_{0}\right)$ the set of all connected components $X_{0}$ of the fixed-point submanifold $X^{T_{0}}$ such that there is $x \in X_{0}$ with $T_{x}=T_{0}$. Set $\mathcal{D}\left(T_{0}\right):=\left\{\pi\left(X_{0}\right), X_{0} \in \widetilde{\mathcal{D}}\left(T_{0}\right)\right\}$. We will see below (assertion 2 of Lemma 2.7) that any element of $\mathcal{D}\left(T_{0}\right)$ is a connected component of $\psi^{-1}\left(\alpha+\mathfrak{t}_{0}^{\perp}\right)$, where $\alpha \in \mathfrak{t}^{*}$ and $\mathfrak{t}_{0}^{\perp}$ denotes the annihilator of $\mathfrak{t}_{0}$ in $\mathfrak{t}^{*}$. Set $\mathcal{D}:=\cup_{T_{0}} \mathcal{D}\left(T_{0}\right)$. The set of divisors $\mathcal{D}$ is the third piece of data we need.

The fourth piece of data is a system of certain elements of $H^{2}(Y, \mathbb{Z})$. To a character $\chi$ of $T$ we assign the coherent sheaf $\mathcal{O}_{\chi}$ on $Y$ defined by

$$
\mathcal{O}_{\chi}(U)=\left\{f \in \mathcal{O}\left(\pi^{-1}(U)\right) \mid t . f=\chi(t) f, \forall t \in T\right\} .
$$

We will see (assertion 3 of Lemma 2.7) that $\mathcal{O}_{\chi}$ is a line bundle for any $\chi$. Let us fix a basis $\chi_{1}, \ldots, \chi_{n}$ in the character group $\mathfrak{X}(T)$ of $T$. Since $Y$ is Stein, we see that the groups $\operatorname{Pic}(Y)$ and $H^{2}(Y, \mathbb{Z})$ are naturally isomorphic. So we get $n$ cohomology classes $c_{i}:=\left[\mathcal{O}_{\chi_{i}}\right] \in H^{2}(Y, \mathbb{Z})$. We remark that $\chi \mapsto\left[\mathcal{O}_{\chi}\right]$ is not a group homomorphism despite for any characters $\chi_{1}, \chi_{2}$ there is a natural map $\mathcal{O}_{\chi_{1}} \otimes \mathcal{O}_{\chi_{2}} \rightarrow \mathcal{O}_{\chi_{1}+\chi_{2}}$.

Finally, we will see (assertion 4 of Lemma 2.7) that there is a holomorphic 2-form $\omega_{0}$ on $Y$ such that $\omega=\pi^{*}\left(\omega_{0}\right)-d \alpha$, where $\alpha$ is a $T$-invariant holomorphic 1-form on $X$ satisfying $\alpha\left(\xi_{*}\right)=H_{\xi}$ for any $\xi \in \mathfrak{t}$; here and below $H_{\xi}=\langle\mu, \xi\rangle$. Moreover, the class $c_{0}$ of $\omega_{0}$ in the second DeRham cohomology group $H_{\mathrm{DR}}^{2}(Y)$ is well-defined. Since $Y$ is Stein, we know that $H_{\mathrm{DR}}^{2}(Y)$ is naturally isomorphic to $H^{2}(Y, \mathbb{C})$, see $[\mathbf{O}]$, Theorem 4.16.

So to any MF Hamiltonian Stein $T$-manifold $X$ we have assigned the 5-tuple $\mathcal{Y}_{X}:=\left(Y, \psi, \mathcal{D},\left(c_{i}\right)_{i=1}^{n}, c_{0}\right)$. Now let $\mathcal{Y}:=\left(Y, \psi, \mathcal{D},\left(c_{i}\right)_{i=1}^{n}, c_{0}\right), \mathcal{Y}^{\prime}:=$ $\left(Y^{\prime}, \psi^{\prime}, \mathcal{D}^{\prime},\left(c_{i}^{\prime}\right)_{i=1}^{n}, c_{0}^{\prime}\right)$ be two 5 -tuples of the indicated form. By a morphism between $\mathcal{Y}, \mathcal{Y}^{\prime}$ we mean an étale holomorphic map $\varphi: Y \rightarrow Y^{\prime}$ satisfying the following conditions:

(a) $\psi=\psi^{\prime} \circ \varphi$.

(b) $\mathcal{D}$ is the set of connected components of $\bigcup_{Y_{0}^{\prime} \in \mathcal{D}^{\prime}} \varphi^{-1}\left(Y_{0}^{\prime}\right)$.

(c) $c_{i}:=\varphi^{*}\left(c_{i}^{\prime}\right), i=\overline{0, n}$.

Let $X, X^{\prime}$ be MF Hamiltonian Stein $T$-manifolds (recall that $T$ is assumed to act effectively on both $\left.X, X^{\prime}\right)$ and $\omega, \omega^{\prime}, \mu, \mu^{\prime}$ be the corresponding symplectic forms and the moment maps. By a Hamiltonian morphism between $X$ and $X^{\prime}$ we mean a holomorphic T-equivariant map $f: X \rightarrow X^{\prime}$ such that $f^{*}\left(\omega^{\prime}\right)=\omega, \mu=\mu^{\prime} \circ f$. Since $\psi, \psi^{\prime}$ are both étale, we easily see that a Hamiltonian morphism $f: X \rightarrow X^{\prime}$ gives rise to a unique morphism $\mathcal{Y}_{f}: \mathcal{Y}_{X} \rightarrow \mathcal{Y}_{X^{\prime}}$ such that $\mathcal{Y}_{f} \circ \pi=\pi^{\prime} \circ \mathcal{Y}_{f}$, where $\pi, \pi^{\prime}$ denote the quotient maps for $X, X^{\prime}$. 
Theorem 1.1. Let $X, X^{\prime}$ be such as above. For any morphism $\varphi: \mathcal{Y}_{X} \rightarrow$ $\mathcal{Y}_{X^{\prime}}$ there is a Hamiltonian morphism $f: X \rightarrow X^{\prime}$ with $\mathcal{Y}_{f}=\varphi$. In particular, $X, X^{\prime}$ are isomorphic if $\mathcal{Y}_{X}, \mathcal{Y}_{X^{\prime}}$ are.

We note that such a morphism $f$ is not necessarily unique. For example, shifting $f$ by an element of $T$, we again get a Hamiltonian morphism with desired properties.

Our next task is to give a characterization of 5 -tuples $\mathcal{Y}$ of the form $\mathcal{Y}_{X}$. We say that a 5-tuple $\mathcal{Y}$ is Delzant if it enjoys the following property:

(Del) Let $y$ be an arbitrary point of $Y, Y_{0}^{1}, \ldots, Y_{0}^{k}$ be all elements of $\mathcal{D}$ containing $y$ and $T_{0}^{1}, \ldots, T_{0}^{k}$ be the corresponding connected onedimensional subgroups of $T$. Then there is a neighborhood $U$ of $y$ in $Y$ such that any element of $\mathcal{D}$ intersecting $U$ is one of $Y_{0}^{i}, i=\overline{1, k}$. Furthermore, the primitive elements of $\mathfrak{X}\left(T_{0}^{i}\right)^{*} \hookrightarrow \mathfrak{X}(T)^{*}, i=\overline{1, k}$, (each of them is defined uniquely up to the multiplication by \pm 1 ) constitute a part of a basis in the lattice $\mathfrak{X}(T)^{*}$.

Theorem 1.2. Suppose a 5-tuple $\mathcal{Y}$ is Delzant. Then there is a MF Hamiltonian Stein $T$-manifold $X$ such that $\mathcal{Y}=\mathcal{Y}_{X}$.

Let us describe the structure of this paper. Section 2 is devoted to some general results concerning MF Hamiltonian Stein $T$-manifolds. Firstly, we recall results of Snow concerning general reductive group actions on Stein manifolds. Then we establish the slice theorem 2.4, which asserts that locally a MF Hamiltonian Stein manifold looks like a model manifold introduced in Example 2.2. Using this theorem we prove some structure results on MF Hamiltonian Stein manifolds, Lemmas 2.6, 2.7. In Section 3 we establish a certain sheaf of groups $\mathcal{A} u t$ on the quotient $Y$ of a MF Hamiltonian Stein manifold $X$. Its sections are Hamiltonian automorphisms that preserve the quotient map. This sheaf is important because it controls gluing of local pieces of MF Hamiltonian manifolds together. The main goal of the section is to determine the sheaf $\mathcal{A}$ ut up to an isomorphism (Lemmas 3.2, 3.3). In Section 4 we prove Theorem 1.1. The most crucial part of its proof is the special case of the identity morphism $\varphi$. To examine this case we consider a natural action of $H^{1}(Y, \mathcal{A} u t)$ on the set of all isomorphism classes of $\mathrm{MF}$ Hamiltonian Stein $T$-manifolds $X$ such that $\mathcal{Y}_{X}=(Y, \psi, \mathcal{D}, \bullet, \bullet)$. Finally, in Section 5 we prove Theorem 1.2. The main idea of the proof is to consider certain local lagrangian sections of the quotient map.

\section{Generalities on MF Hamiltonian Stein $T$-manifolds}

Until a further notice $T$ denotes an algebraic torus $\left(\mathbb{C}^{\times}\right)^{n}$ and $X$ is a Stein $T$-manifold.

By $[\mathbf{S}]$, there is a Stein space $Y$ and a surjective $T$-invariant holomorphic map $\pi: X \rightarrow Y$ satisfying the following conditions: 
(A) $U \subset Y$ is open iff $\pi^{-1}(U)$ is,

(B) $\pi^{*}(\mathcal{O}(U))=\mathcal{O}\left(\pi^{-1}(U)\right)^{T}$ for any open subset $U \subset Y$.

Such $Y, \pi$ have the following universal property: for any Stein space $Z$ and a $T$-equivariant holomorphic map $\rho: X \rightarrow Z$ there is a unique $T$-equivariant holomorphic map $\rho: Y \rightarrow Z$ such that $\rho=\rho \circ \pi$. Therefore $Y$ is called the categorical quotient for the action $T: X$ and $\pi$ is called the quotient map. It is known, see $[\mathbf{S}]$, that any fiber of $\pi$ contains a unique closed $T$-orbit.

In the sequel we will need the following notion.

Definition 2.1. A subset $X_{0} \subset X$ is called saturated if it consists of fibers of the quotient map, that is $X_{0}=\pi^{-1}\left(\pi\left(X_{0}\right)\right)$.

If $X_{0}$ is open and saturated, then $\pi\left(X_{0}\right)$ is open.

Now we quote the Snow slice theorem. Let $x \in X$ be a point with closed $T$-orbit. Let $T_{0}$ denote the stabilizer $T_{x}$ of $x$ in $T$ and $V$ denote the slice $T_{0}$-module $T_{x} X / \mathfrak{t}_{*} x$. Here and below $\mathfrak{t}_{*} x$ denotes the tangent space to the orbit $T x$. Then there is an open saturated neighborhood $U$ of $x$ in $X$ and an open $T_{0}$-stable neighborhood $U_{0}$ of 0 in $V$ such that $U$ is $T$-equivariantly isomorphic to the homogeneous bundle $T * T_{0} U_{0}$. By definition, $T *_{T_{0}} U_{0}$ is the orbit space for the action $T_{0}: T \times U_{0}$ given by $t_{0} \cdot(t, u)=\left(t t_{0}^{-1}, t_{0} u\right), t \in$ $T, u \in U, t_{0} \in T_{0}$; it is easy to see that $T *_{T_{0}} U_{0}$ is a Stein manifold. Let $\pi_{0}$ denote the quotient map for the action $T_{0}: V$. From property (B) above it follows that $\pi(U) \cong \pi_{0}\left(U_{0}\right)$ and the quotient $\pi: U \rightarrow \pi(U)$ is identified with $T *_{T_{0}} U_{0} \rightarrow \pi_{0}\left(U_{0}\right)$. Since $U$ is saturated, we see that $U_{0}$ is also saturated (w.r.t. $T_{0}$ ).

Now we would like to produce some examples of MF Hamiltonian Stein $T$-manifolds.

Example 2.2. Fix a connected subgroup $T_{0} \subset T$, characters $\chi_{1}, \ldots, \chi_{k}$ of $T_{0}$ forming a basis of $\mathfrak{X}\left(T_{0}\right)$, and $\lambda \in \mathfrak{t}^{*}$. To these data we assign an MF Hamiltonian Stein manifold $X$ as follows. Let $V$ be a vector space with a basis $v_{1}, \ldots, v_{k}$. Define the linear action $T_{0}: V$ by $t . v_{i}=\chi_{i}(t) v_{i}, i=$ $\overline{1, k}, t \in T_{0}$. Let $X_{0}$ be the homogeneous vector bundle $T *_{T_{0}} V$. There is a subtorus $T_{1} \subset T$ such that $T_{1} \times T_{0}=T$. For any such $T_{1}$ we have $X_{0}=$ $T_{1} \times V$. Choose a basis $\theta_{1}, \ldots, \theta_{l}, l=n-k$, in $\mathfrak{X}\left(T_{1}\right)$, set $\beta_{i}:=\frac{d \theta_{i}}{\theta_{i}}=$ $d\left(\ln \theta_{i}\right)$. One can regard $\beta_{1}, \ldots, \beta_{l}$ as a basis in $\mathfrak{t}_{1}^{*}$. Let $\beta^{i}, i=\overline{1, l}$, be the basis in $\mathfrak{t}_{1}$ dual to $\beta_{i}$. Further, let $v^{1}, \ldots, v^{k}$ be the basis of $V^{*}$ dual to $v_{1}, \ldots, v_{k}$. Set $X:=T^{*} X_{0}$. We can consider $\theta_{i}, \beta^{i}, v_{j}, v^{j}$ as functions on $X$ and these functions form a coordinate system. The manifold $X$ has the natural symplectic form $\omega=-d \alpha$, where $\alpha:=\sum_{i=1}^{l} \beta^{i} d \ln \theta_{i}+\sum_{i=1}^{k} v_{i} d v^{i}$. The form $\alpha$ is $T$-invariant and the action $T: X$ is Hamiltonian with moment map $\mu(x)=\lambda+\sum_{i=1}^{l} \beta^{i}(x) \beta_{i}+\sum_{i=1}^{k} v_{i}(x) v^{i}(x) d\left(\ln \chi_{i}\right)$. One can easily check that $\alpha, \mu$ do not depend on choices we have made (i.e., the choices of $\left.v_{i}, T_{1}, \theta_{j}\right)$. We also note that the action $T: X$ is effective and $\alpha\left(\xi_{*}\right)=H_{\xi}$. 
Let us check that the 5-tuple $\mathcal{Y}_{X}$ is well-defined and compute it. We have $Y \cong \mathbb{C}^{n}, \beta^{1}, \ldots, \beta^{l}, v_{1} v^{1}, \ldots, v_{k} v^{k}$ being coordinates. The map $\psi$ is identical. We easily get $\mathcal{D}=\left\{D_{i}, i=\overline{1, k}\right\}$, where $D_{i}$ is the affine hyperplane of the form $v_{i} v^{i}=$ const. containing $\lambda$.

Choose $\chi \in \mathfrak{X}(T), \chi=\sum_{i=1}^{l} a_{i} \theta_{i}+\sum_{i=1}^{k} b_{i} \chi_{i}$. Then $\mathcal{O}_{\chi}$ is a trivial line bundle on $Y$ generated by $\left(\prod_{i=1}^{l} \theta_{i}^{a_{i}}\right)\left(\prod_{i=1}^{k} u_{i}^{\left|b_{i}\right|}\right)$, where $u_{i}=v_{i}$ if $b_{i} \geqslant 0$ and $v^{i}$ otherwise. So $c_{1}, \ldots, c_{n}$ are well-defined and equal to zero for any choice of a basis in $\mathfrak{X}(T)$.

Since $\omega$ is exact and $H^{2}(Y, \mathbb{C})=0$, we see that $c_{0}$ in this case is welldefined and equals 0 .

We remark that the 5-tuple $\mathcal{Y}_{X}$ is Delzant.

We call $X$ a model Hamiltonian $T$-manifold associated with $T_{0}, \lambda$, $\chi_{1}, \ldots, \chi_{k}$. By a base point in $X$ we mean any point from a unique closed $T$-orbit in $X_{0}$ (i.e., any point $x \in X_{0}$ with $v^{1}(x)=\ldots=v^{k}(x)=0$ ).

Definition 2.3. Let $X_{1}, X_{2}$ be MF Hamiltonian Stein $T$-manifolds, $x_{1} \in$ $X_{1}, x_{2} \in X_{2}$ be points with closed $T$-orbits. We say that the pairs $\left(X_{1}, x_{1}\right)$, $\left(X_{2}, x_{2}\right)$ are locally equivalent if there are saturated open neighborhoods $U_{i}, i=1,2$, of $x_{i}$ in $X_{i}$ and a Hamiltonian isomorphism $\varphi: U_{1} \rightarrow U_{2}$ such that $\varphi\left(x_{1}\right)=x_{2}$.

Till the end of the section $X$ is an arbitrary MF Hamiltonian Stein T-manifold.

Theorem 2.4 (symplectic slice). Let $x \in X$ be a point with closed orbit, $T_{0}=T_{x}, \lambda=\mu(x)$. Then the following assertions hold:

(1) $T_{0}$ is connected, and the set of weights of $T_{0}$ in $T_{x} X$ has the form $\left\{0, \chi_{1}, \ldots, \chi_{k},-\chi_{1}, \ldots,-\chi_{k}\right\}$, where $\chi_{1}, \ldots, \chi_{k}$ is a basis of the character group $\mathfrak{X}\left(T_{0}\right)$.

(2) Let $X^{\prime}$ be a model Hamiltonian $T$-manifold associated with $T_{0}, \lambda$, $\chi_{1}, \ldots, \chi_{k}$ and $x^{\prime}$ its base point. Then the pairs $(X, x)$ and $\left(X^{\prime}, x^{\prime}\right)$ are locally equivalent.

Proof. Note that $T_{x} X$ is a symplectic $T_{0}$-module. There is a trivial isotropic $T_{0}$-submodule $\mathfrak{t}_{*} x \subset T_{x} X$. Therefore $T_{x} X /\left(\mathfrak{t}_{*} x\right)^{<}$, where ${ }^{<}$denotes the skeworthogonal complement, is also a trivial $T_{0}$-module. Set $V:=\left(\mathfrak{t}_{*} x\right)^{<} / \mathfrak{t}_{*} x$. This is a symplectic $T_{0}$-module of dimension $\operatorname{dim} X-2 \operatorname{dim} T x=2 \operatorname{dim} T_{0}$. By Snow's slice theorem, the action $T_{0}: V$ is effective. It follows that the weights of $T_{0}^{\circ}$ in $V$ have the form $\chi_{1}, \ldots, \chi_{k},-\chi_{1}, \ldots,-\chi_{k}$, where $\chi_{1}, \ldots, \chi_{k}$ form a basis of $\mathfrak{X}\left(T_{0}^{\circ}\right)$. Therefore $T_{0}^{\circ}$ is a maximal torus in $\operatorname{Sp}(V)$ whence $T_{0}=T_{0}^{\circ}$. So assertion (1) is proved.

In view of the Snow slice theorem, the proof of assertion (2) is completely analogous to that of the symplectic slice theorem from $[\mathbf{L}]$ (where it was proved for Hamiltonian reductive group actions on affine algebraic varieties). 
Remark 2.5. Now let $X^{1}, X^{2}$ be model Hamiltonian $T$-manifolds corresponding to the data $\left(T_{0}^{1}, \lambda^{1}, \chi_{1}^{1}, \ldots, \chi_{k_{1}}^{1}\right),\left(T_{0}^{2}, \lambda^{2}, \chi_{1}^{2}, \ldots, \chi_{k_{2}}^{2}\right)$ and $x^{1}, x^{2}$ be the corresponding base points. Then the pairs $\left(X^{1}, x^{1}\right),\left(X^{2}, x^{2}\right)$ are locally equivalent iff the following conditions are satisfied:

(1) $T_{0}^{1}=T_{0}^{2}$ whence $k_{1}=k_{2}$.

(2) $\lambda^{1}=\lambda^{2}$.

(3) After reordering $\chi_{1}^{2}, \ldots, \chi_{k_{2}}^{2}$, we get $\chi_{i}^{1}= \pm \chi_{i}^{2}, i=\overline{1, k_{1}}$.

Below we will often use a certain open submanifold of $X$ associated with a "general enough" element $\zeta \in \mathfrak{X}(T) \otimes \mathbb{R}$.

We say that $\zeta \in \mathfrak{X}(T) \otimes \mathbb{R}$ is general for $X$ if $\zeta$ is nonzero on the Lie algebra of $T_{x}$ for any $x \in X$. Since the set of all $T_{x}$ is countable, we see that such $\zeta$ does exist. By $X^{\zeta}$ we denote the subset of $X$ consisting of all points $x$ satisfying the following two conditions:

(1) $T_{x}=\{1\}$.

(2) Let $\tau: \mathbb{C}^{\times} \rightarrow T$ be a one-parameter subgroup. If $\lim _{t \rightarrow 0} \tau(t) . x$ exists in $X$, then $\langle\zeta, \tau\rangle>0$ (we note that the l.h.s of the previous inequality is always nonzero because $\operatorname{im} \tau$ lies in the stabilizer of $\left.\lim _{t \rightarrow 0} \tau(t) . x\right)$.

The following lemma describes some properties of the subset $X^{\zeta} \subset X$.

Lemma 2.6. (1) If $X_{0}$ is an open saturated Stein subset of $X$, then $\left(X_{0}\right)^{\zeta}=X^{\zeta} \cap X_{0}$.

(2) The set $X^{\zeta}$ is open in $X$.

(3) For any $y \in Y$ the intersection $X^{\zeta} \cap \pi^{-1}(y)$ is a single T-orbit. In particular, the map $\left.\pi\right|_{X^{\zeta}}: X^{\zeta} \rightarrow Y$ is surjective.

(4) The map $\left.\pi\right|_{X \zeta}: X^{\zeta} \rightarrow Y$ is a locally trivial principal T-bundle whence the quotient map for the action $T: X^{\zeta}$.

Proof. Since $\pi^{-1}(\pi(x)) \subset X$ is a closed subset, for a one parameter subgroup $\tau$ the following three conditions are equivalent:

(1) $\lim _{t \rightarrow 0} \tau(t) . x$ exists in $X$.

(2) $\lim _{t \rightarrow 0} \tau(t) \cdot x$ exists in $\pi^{-1}(\pi(x))$.

(3) $\lim _{t \rightarrow 0} \tau(t) . x$ exists in $X^{0}$.

Hence the first assertion.

Using the Snow slice theorem we reduce assertions (2), (3) to the case when $X$ is a model Hamiltonian manifold (we do not need a symplectic structure here). Let $T_{0}, \chi_{1}, \ldots, \chi_{k}, \lambda, \theta_{i}, \beta^{i}, v_{j}, v^{j}$ be such as in Example 2.2. Write the decomposition $\zeta=\sum_{i=1}^{l} a_{i} \theta_{i}+\sum_{i=1}^{k} b_{i} \chi_{i}$. The condition that $\zeta$ is general implies that all $b_{i}$ are nonzero. Thanks to Remark 2.5, we may assume that all $b_{i}$ are positive. In this case one checks directly that $X^{\zeta}=$ $\left\{x \in X \mid v_{i}(x) \neq 0, i=\overline{1, k}\right\}$ and assertions (2), (3) follow. 
Assertion (4) follows from the observation that there is a holomorphic slice $S$ for the action $T: X^{\zeta}$ at any point of $X^{\zeta}$ and the restriction of $\pi$ to $S$ is an open embedding $S \hookrightarrow Y$.

Lemma 2.7. (1) $Y$ is smooth and $\psi$ is étale.

(2) Let $T_{0}$ be a one-dimensional connected subgroup of $T$ and $Y_{0} \in \mathcal{D}\left(T_{0}\right)$. Then there is $\alpha \in \mathfrak{t}^{*}$ such that $Y_{0}$ is a connected component of $\psi^{-1}(\alpha+$ $\left.\mathfrak{t}_{0}^{\perp}\right)$.

(3) For any $\chi \in \mathfrak{X}(T)$ the sheaf $\mathcal{O}_{\chi}$ is a line bundle.

(4) There is a 2-form $\omega_{0} \in \Omega^{2}(Y)$ and a $T$-invariant one-form $\alpha \in \Omega^{1}(X)$ such that $\omega=\pi^{*}\left(\omega_{0}\right)-d \alpha$, and $\alpha\left(\xi_{*}\right)=H_{\xi}$ for any $\xi \in \mathfrak{t}$. Moreover, the class of $\omega_{0}$ in $H_{D R}^{2}(Y)$ does not depend on the choice $\omega_{0}, \alpha$.

(5) The 5-tuple $\mathcal{Y}_{X}$ is well-defined and Delzant.

In the proof below we will need the following elementary fact.

Lemma 2.8. Let $y \in Y$. Then the manifold $\pi^{-1}\left(U_{0}\right)$ is Stein for any open Stein neighborhood $U_{0}$ of $y$ in $Y$.

Proof. Note that $\pi^{-1}\left(U_{0}\right)$ is the fiber product $U_{0} \times_{Y} X$. Being a product of two Stein manifolds, $U_{0} \times X$ is Stein. Since $\pi^{-1}\left(U_{0}\right)=U_{0} \times_{Y} X$ is closed in $U_{0} \times X$, we are done.

Proof of Lemma 2.7. Everything but assertion (4) follows directly from Theorem 2.4 and Example 2.2. Let us prove assertion (4).

Let us check that $\left[\omega_{0}\right] \in H_{D R}^{2}(Y)$ is unique if $\omega_{0}$ exists. Let $\omega=\pi^{*}\left(\omega_{0}^{1}\right)-$ $d \alpha^{1}=\pi^{*}\left(\omega_{0}^{2}\right)-d \alpha^{2}$ be two representations of the required form. Then $\alpha^{0}:=\alpha^{1}-\alpha^{2}$ is a $T$-invariant form such that $\alpha^{0}\left(\xi_{*}\right)=0$. Choose a general for $X$ element $\zeta \in \mathfrak{X}(T) \otimes_{\mathbb{Z}} \mathbb{R}$. By assertion 4 of Lemma 2.6, the map $\pi: X^{\zeta} \rightarrow Y$ is a principal $T$-bundle. It follows that there is $\alpha_{0} \in \Omega^{1}(Y)$ such that $\alpha^{0}=\pi^{*}\left(\alpha_{0}\right)$ on $X^{\zeta}$ and thence on the whole manifold $X$. So $\pi^{*}\left(\omega_{0}^{1}-d \alpha_{0}\right)=\pi^{*}\left(\omega_{0}^{2}\right)$ whence $\omega_{0}^{1}-d \alpha_{0}=\omega_{0}^{2}$.

So it remains to prove that there exist $\omega_{0}, \alpha$ with the required properties.

It follows from Example 2.2 and Lemma 2.8 that there exists an open covering $Y=\cup_{i \in I} Y_{i}$ satisfying the following conditions:

(1) Both $Y_{i}$ and $\pi^{-1}\left(Y_{i}\right)$ are Stein.

(2) $\left.\omega\right|_{\pi^{-1}\left(Y_{i}\right)}=-d \alpha_{i}$, where $\alpha_{i}$ is a holomorphic $T$-invariant 1-form on $\pi^{-1}\left(Y_{i}\right)$ such that $\alpha_{i}\left(\xi_{*}\right)=H_{\xi}$.

For a finite subset $J \subset I$ set $Y_{J}:=\cap_{j \in J} Y_{j}$.

Set $\alpha_{i j}:=\alpha_{i}-\alpha_{j}$. This is a $T$-invariant holomorphic 1-form on $\pi^{-1}\left(Y_{i j}\right)$ with $\alpha_{i j}\left(\xi_{*}\right)=0$. It follows that there is a (unique) holomorphic 1-form $\gamma_{i j}$ on $Y_{i j}$ such that $\alpha_{i j}=\pi^{*}\left(\gamma_{i j}\right)$. The collection $\left(\gamma_{i j}\right)$ is a 1-cocycle in $\Omega^{1}$. Since $Y$ is Stein, we see that there are holomorphic 1-forms $\gamma_{i}$ on $Y_{i}$ such that $\gamma_{i j}=\gamma_{i}-\gamma_{j}$. Further, $0=d \alpha_{i j}=\pi^{*}\left(d \gamma_{i j}\right)$, whence $d \gamma_{i}=d \gamma_{j}$ on $Y_{i j}$. 
Set $\omega_{0}:=d \gamma_{i}$ on $Y_{i}$. Then $\omega-\omega_{0}$ coincides with $d\left(\alpha_{i}-\pi^{*}\left(\gamma_{i}\right)\right)$ on $\pi^{-1}\left(Y_{i}\right)$. Since $\alpha_{i}-\pi^{*}\left(\gamma_{i}\right)=\alpha_{j}-\pi^{*}\left(\gamma_{j}\right)$ on $\pi^{-1}\left(Y_{j}\right)$, we may set $\alpha=\alpha_{i}-\pi^{*}\left(\gamma_{i}\right)$.

Remark 2.9. Let $\mathcal{Y}_{X}=\left(Y, \psi, \mathcal{D},\left(c_{i}\right)_{i=1}^{n}, c_{0}\right)$. Let us show how to recover the structure of $X$ in a neighborhood of a point $x \in X$ with closed orbit. Let $D_{1}, \ldots, D_{k}$ be all elements of $\mathcal{D}$ containing $\pi(x)$. For each $i$ there is a unique affine hyperplane $\Gamma_{i} \subset \mathfrak{t}^{*}$ containing $\psi\left(D_{i}\right)$. Let $\eta_{i}, i=\overline{1, k}$, be a nonzero element from $\mathfrak{t}$ lying in the annihilator of the (linear) hyperplane associated with $\Gamma_{i}$. Then the Lie algebra of $T_{0}$ is spanned by $\eta_{1}, \ldots, \eta_{k}$, the character $\chi_{i}, i=\overline{1, k}$ is a primitive element of $\mathfrak{X}\left(T_{0}\right)$ annihilating all $\eta_{j}$ with $j \neq i$ (a character $\chi_{i}$ is determined uniquely up to changing the sign), and $\lambda=\psi(x)$.

In the sequel we will need the following proposition that is a special case of Theorem in Section 1 of $[\mathbf{H M P}]$.

Proposition 2.10. Let $X$ be a complex $T$-manifold such that the action $T: X$ is effective, $Y$ a Stein manifold, and $\pi: X \rightarrow Y$ a holomorphic $T$ invariant map. Suppose that there is an open covering $Y_{i}, i \in I$, of $Y$ by Stein submanifolds such that $\pi^{-1}\left(Y_{i}\right)$ is a Stein T-manifold and $\pi: \pi^{-1}\left(Y_{i}\right) \rightarrow Y_{i}$ is the quotient map. Then $X$ is Stein and $\pi: X \rightarrow Y$ is the quotient map.

\section{The sheaf $\mathcal{A u t}$}

Let $T, X, \pi, \mathcal{Y}_{X}=\left(Y, \psi, \mathcal{D},\left(c_{i}\right)_{i=1}^{n}, c_{0}\right)$ be such as above. The goal of this section is to study the sheaf of groups $\mathcal{A} u t^{X}$ on $Y$ defined as follows: the group $\mathcal{A} u t^{X}(U)$ consists of all Hamiltonian morphisms of $\pi^{-1}(U)$ preserving $\pi$. This sheaf plays a crucial role in the subsequent development, compare with the proof of the uniqueness part of the Delzant theorem in $[\mathbf{W}]$.

At first, we construct a certain morphism of sheafs $\mathcal{O}_{Y} \rightarrow \mathcal{A} u t^{X}$.

To any function $f \in \mathcal{O}(U)$, where $U$ is an open subset of $X$, we assign its skew-gradient $v(f)$ by

$$
\omega_{x}(v(f), \eta)=\left\langle\eta, d_{x} f\right\rangle, x \in U, \eta \in T_{x} X .
$$

Exponentiating the vector field $v(f)$ we get the local one-parameter subgroup $t \mapsto \exp (t v(f)), t \in \mathbb{C}$, of holomorphic automorphisms of $X$. Since $v(f) \cdot \omega=d \iota_{v(f)} \omega+\iota_{v(f)} d \omega\left(\iota_{v(f)}\right.$ denotes the contraction with $\left.v(f)\right)$, we see that $v(f)$ annihilates $\omega$. So $\exp (t v(f))$ preserves $\omega$.

Lemma 3.1. Let $U$ be an open subset of $Y$ and $f \in \mathcal{O}(U) \cong \mathcal{O}\left(\pi^{-1}(U)\right)^{T}$. Then the map $t \mapsto \exp (2 \pi i t v(f))$ is a well-defined homomorphism $\mathbb{C} \rightarrow$ $\mathcal{A} u t^{X}(U)$ such that the corresponding action $\mathbb{C}: \pi^{-1}(U)$ is holomorphic and its velocity vector field coincides with $2 \pi i v(f)$.

Proof. Define two constant sheafs $\mathfrak{t}_{Y}, T_{Y}$ on $Y$ with fibers $\mathfrak{t}, T$, respectively. There is the natural epimorphism $\mathfrak{t}_{Y} \rightarrow T_{Y}, \xi \mapsto \exp (2 \pi i \xi)$. Also there is the natural action $T_{Y}(U): \pi^{-1}(U), \varphi \cdot x=\varphi(\pi(x)) x$ by holomorphic $T$-equivariant automorphisms preserving $\pi$. 
At first, suppose that the action $T: \pi^{-1}(U)$ is free. Note that the vector field $v(f)$ is $T$-invariant and tangent to all $T$-orbits (the latter stems from $\omega\left(v(f), \xi_{*}\right)=0$ for any $\left.\xi \in \mathfrak{t}\right)$. So we may consider $v(f)$ as a section of $\mathfrak{t}_{Y}$. Applying the exponential map to $v(f)$, we get the one-parameter subgroup $\exp (2 \pi i t v(f))$ of $T_{Y}(U)$. So we get the action $\mathbb{C}: \pi^{-1}(U)$, whose velocity vector field is $2 \pi i v(f)$. Since $v(f)$ is a Hamiltonian vector field, this action preserves $\omega$. Finally, by definition, the action preserves $\pi$ and thus also $\mu$.

Consider the general case. Choose general $\zeta \in \mathfrak{X}(T) \otimes_{\mathbb{Z}} \mathbb{R}$. Since the actions of $T_{Y}(U)$ and $T$ commute, it follows from the definition of $X^{\zeta}$ that $\pi^{-1}(U) \cap X^{\zeta}$ is stable with respect to the action $T_{Y}(U): \pi^{-1}(U)$. The action $\mathbb{C}: X^{\zeta} \cap \pi^{-1}(U)$ constructed above is factorized through a homomorphism $\mathbb{C} \rightarrow T_{Y}(U)$. Since $T_{Y}(U)$ acts on $\pi^{-1}(U)$ by holomorphic automorphisms, we see that the action $\mathbb{C}: \pi^{-1}(U) \cap X^{\zeta}$ can be extended to an action by holomorphic symplectomorphisms on the whole set $\pi^{-1}(U)$.

So we have the sheaf morphism $\mathcal{O}_{Y} \rightarrow \mathcal{A} u t^{X}$ given by

$$
f \mapsto \exp (2 \pi i v(f)) .
$$

Lemma 3.2. The morphism of sheafs (3.1) is surjective.

Proof. Again, choose an element $\zeta \in \mathfrak{X}(T) \otimes_{\mathbb{Z}} \mathbb{R}$ general for $X$ and an open subset $U \subset Y$. We get a natural embedding $\mathcal{A} u t^{X}(U) \hookrightarrow \mathcal{A} u t^{X^{\zeta}}(U)$ induced by the restriction to $X^{\zeta} \cap \pi^{-1}(U)$. This embedding is compatible with morphisms $\mathcal{O}_{Y} \rightarrow \mathcal{A} u t^{X}, \mathcal{O}_{Y} \rightarrow \mathcal{A} u t^{X^{\zeta}}$. So we may replace $X$ with $X^{\zeta}$ and assume that the action $T: X$ is free. Since the question is local, we may assume $X=T^{*}(T)$ and $U$ is given by (in the notation of Example 2.2) $\left|\beta^{i}\right|<1, i=\overline{1, n}$. We need to prove that the map $\mathcal{O}(U) \rightarrow \mathcal{A} u t^{X}(U)$ is surjective.

The group $\mathcal{A} u t^{X}(U) \hookrightarrow T_{Y}(U)$ consists of all holomorphic maps $\Phi=$ $\left(\Phi_{1}, \ldots, \Phi_{n}\right): U \rightarrow T$ such that the map $(t, y) \mapsto(\Phi(y) t, y)$ preserves $\omega$. Fix a basis $\theta_{1}, \ldots, \theta_{n}$ of $\mathfrak{X}(T)$. Since $U$ is simply connected, there is a holomorphic map $\varphi=\left(\varphi_{1}, \ldots, \varphi_{n}\right): U \rightarrow \mathfrak{t} \operatorname{such}$ that $\Phi=\exp (2 \pi i \varphi)$. A direct computation shows that the map $(t, y) \mapsto(\Phi(y) t, y)$ preserves $\omega$ iff $\sum_{i=1}^{n} \frac{d \Phi_{i}}{\Phi_{i}} \wedge d y_{i}=0$. The latter is equivalent to the system of equations

$$
\frac{\partial \varphi_{i}}{\partial \beta^{j}}=\frac{\partial \varphi_{j}}{\partial \beta^{i}}, \quad i, j=\overline{1, n} .
$$

By the Dolbeaux lemma, there is $f \in \mathcal{O}(U)$ such that $\varphi_{i}=\frac{\partial f}{\partial \beta^{i}}$. It follows that $v(f)=\varphi$. The homomorphism $\mathcal{O}(U) \rightarrow \mathcal{A} u t^{X}(U)$ maps $f$ to $\Phi$.

Lemma 3.3. The kernel of the epimorphism (3.1) is $\mathbb{C} \oplus \mathfrak{X}(T)^{*}$ (where $\mathbb{C}$ denotes the sheaf of constant functions and $\mathfrak{X}(T)^{*}$ the sheaf of $\left.H_{\xi}, \xi \in \mathfrak{X}(T)^{*}\right)$. 
Proof. Again, it is enough to prove this lemma for $X=T^{*}(T)$, where it is checked directly.

Finally, $\mathcal{A} u t^{X} \cong \mathcal{O}_{Y} /\left(\mathbb{C} \oplus \mathfrak{X}(T)^{*}\right)$. In particular, $\mathcal{A} u t^{X}$ depends only on $Y$ and $\psi: Y \rightarrow \mathfrak{t}^{*}$, so we write $\mathcal{A} u t$ instead of $\mathcal{A} u t^{X}$.

Corollary 3.4. $H^{j}(Y, \mathcal{A} u t)=H^{j+1}\left(Y, \mathbb{C} \oplus \mathfrak{X}(T)^{*}\right)$.

Proof. Since $Y$ is Stein, we have $H^{j}\left(Y, \mathcal{O}_{Y}\right)=0$ for $j>0$. It remains to consider the long exact sequence associated with $0 \rightarrow \mathbb{C} \oplus \mathfrak{X}(T)^{*} \rightarrow \mathcal{O}_{Y} \rightarrow$ $\mathcal{A} u t \rightarrow 0$.

\section{Uniqueness}

The goal of this section is to prove Theorem 1.1. Throughout this section $X$ is an MF Hamiltonian Stein $T$-manifold and $\mathcal{Y}_{X}=\left(Y, \psi, \mathcal{D},\left(c_{i}\right)_{i=1}^{n}, c_{0}\right)$ the corresponding 5-tuple.

Denote by $\mathbb{X}$ the set of all isomorphism classes of multiplicity free Hamiltonian Stein $T$-manifolds with 5 -tuples of the form $(Y, \psi, \mathcal{D}, \bullet, \bullet)$. Two such Hamiltonian $T$-manifolds $X, X^{\prime}$ are supposed to be isomorphic if there is a Hamiltonian isomorphism $\iota: X \rightarrow X^{\prime}$ such that $\pi^{\prime} \circ \iota=\pi$, where $\pi, \pi^{\prime}$ are the quotient maps for $X, X^{\prime}$.

There is a natural action of $H^{1}(Y, \mathcal{A} u t)$ on $\mathbb{X}$, which we describe now.

Choose $c \in H^{1}(Y, \mathcal{A} u t)$. Let $Y_{i}, i \in I$, be an open covering and $\varphi_{i j} \in$ $\mathcal{A} u t\left(Y_{i j}\right)$, where $Y_{i j}:=Y_{i} \cap Y_{j}$, be a 1-cocycle representing $c$. Set

$$
X^{\prime}:=\coprod_{i \in I} \pi^{-1}\left(Y_{i}\right) / \sim
$$

where points $x_{i} \in \pi^{-1}\left(Y_{i}\right), x_{j} \in \pi^{-1}\left(Y_{j}\right)$ are equivalent if they both lie in $\pi^{-1}\left(Y_{i j}\right)$ and $x_{i}=\varphi_{i j}\left(x_{j}\right)$ (the equality of points in $X$ ). Since $\left(\varphi_{i j}\right)$ is a 1 -cocycle, we see that $\sim$ is a genuine equivalence relation. Clearly, $X^{\prime}$ has a unique structure of a Hamiltonian $T$-manifold such that the embedding $\pi^{-1}\left(Y_{i}\right) \rightarrow X^{\prime}$ is a Hamiltonian morphism. It follows from Proposition 2.10 that $X^{\prime}$ is Stein.

It is clear that if $\varphi_{i j}^{\prime}$ is another 1-cycle and $X^{\prime \prime}$ is obtained by applying $\varphi_{i j}^{\prime}$ to $X^{\prime}$, then $X^{\prime \prime}$ is obtained from $X$ by applying $\varphi_{i j}^{\prime} \varphi_{i j}$. Now suppose $\varphi_{i j}$ is a 1-coboundary, that is, there is a 1-cochain $f_{i} \in \mathcal{A} u t\left(Y_{i}\right)$ with $\varphi_{i j}=f_{i} f_{j}^{-1}$. Then there is the isomorphism $X \rightarrow X^{\prime}$ given by $f_{i}$ on $\pi^{-1}\left(Y_{i}\right)$. So $X^{\prime}$ depends up to isomorphism only on $c$ and we write $X_{c}$ for $X^{\prime}$. Also we note that $X_{c_{1} c_{2}}=\left(X_{c_{1}}\right)_{c_{2}}$, so we do have an action of $H^{1}(Y, \mathcal{A} u t)$ on $\mathbb{X}$.

The following proposition is the main property of this action.

Proposition 4.1. Suppose $\mathbb{X}$ is nonempty. Then the action of $H^{1}(Y, \mathcal{A} u t)$ on $\mathbb{X}$ is free and transitive. Further, for any $\left(c_{i}^{\prime}\right)_{i=0}^{n} \in H^{2}\left(\mathbb{C} \oplus \mathfrak{X}(T)^{*}\right)$ there is a unique element $X^{\prime} \in \mathbb{X}$ with $\mathcal{Y}_{X}=\left(Y, \psi, \mathcal{D},\left(c_{i}^{\prime}\right)_{i=1}^{n}, c_{0}^{\prime}\right)$. 
The proof of this proposition will be given at the end of the section after two auxiliary lemmas. Now we are going to derive Theorem 1.1 from Proposition 4.1.

Proof of Theorem 1.1. Let $X^{\prime}$ be an MF Hamiltonian Stein $T$-manifold and $\varphi: \mathcal{Y}=\left(Y, \psi, \mathcal{D},\left(c_{i}\right)_{i=1}^{n}, c_{0}\right) \rightarrow \mathcal{Y}_{X^{\prime}}=\left(Y^{\prime}, \psi^{\prime}, \mathcal{D}^{\prime},\left(c_{i}^{\prime}\right)_{i=1}^{n}, c_{0}^{\prime}\right)$ be an arbitrary morphism of 5-tuples. Set $X=Y \times_{Y^{\prime}} X^{\prime}$ and let $\widetilde{\psi}$ denote the map $X \rightarrow X^{\prime}$ arising from the Cartesian square. There is a unique structure of a Hamiltonian $T$-manifold on $X$ such that $\widetilde{\psi}$ is a Hamiltonian morphism. Being a closed submanifold in $Y \times X^{\prime}$, the manifold $X$ is Stein. The natural map $\pi: X \rightarrow Y$ is the quotient map. It is clear from construction that $\mathcal{Y}_{X}=\mathcal{Y}$.

The previous construction reduces the proof of the theorem to the case $\varphi=i d$. This case stems from Proposition 4.1.

Let $X^{\prime}$ be constructed from $X$ by applying $c \in H^{1}(Y, \mathcal{A} u t), \pi^{\prime}$ denote the quotient map $X^{\prime} \rightarrow Y$, and $\mathcal{Y}_{X^{\prime}}=\left(Y, \psi^{\prime}, \mathcal{D}^{\prime},\left(c_{i}^{\prime}\right)_{i=1}^{n}, c_{0}^{\prime}\right)$. It is clear from the construction of $X^{\prime}$ that $\psi^{\prime}=\psi, \mathcal{D}^{\prime}=\mathcal{D}$. Let us examine the behavior of $c_{i}^{\prime}$. We recall that $H^{1}(Y, \mathcal{A} u t) \cong H^{2}\left(X, \mathbb{C} \oplus \mathfrak{X}(T)^{*}\right)$ (Corollary 3.4).

Recall also that there is a natural embedding of sheafs $\mathcal{A} u t \hookrightarrow T_{Y}$, see the proof of Lemma 3.1. So one can consider the homomorphism $\varphi \mapsto\langle\chi, \varphi\rangle$ from $\mathcal{A}$ ut to the constant sheaf with fiber $\mathbb{C}^{\times}$.

Lemma 4.2. Choose $\chi \in \mathfrak{X}(T)$ and let $c_{\chi}, c_{\chi}^{\prime}$ be the classes of the line bundles $\mathcal{O}_{\chi}, \mathcal{O}_{\chi}^{\prime}$ corresponding to $X, X^{\prime}$ in $H^{2}(Y, \mathbb{C})$. Then $c_{\chi}^{\prime}=c_{\chi}+\langle\chi, c\rangle$.

Proof. Let $f_{i j}$ denote the transition functions for the sheaf $\mathcal{O}_{\chi}$ on $Y$. From the construction of the isomorphism $H^{1}(Y, \mathcal{A} u t) \cong H^{2}\left(Y, \mathbb{C} \oplus \mathfrak{X}(T)^{*}\right)$ it follows that we need to check that $f_{i j}\left\langle\chi, \varphi_{i j}\right\rangle$ is a system of transition functions for $\mathcal{O}_{\chi}^{\prime}$.

Indeed, let $\mathcal{O}^{\prime}$ be the line bundle on $Y$ with transition functions $f_{i j}\left\langle\chi, \varphi_{i j}\right\rangle$. Let $T$ act on sections of $\mathcal{O}^{\prime}$ by $\chi$. We may assume that $\mathcal{O}^{\prime}$ is trivialized over each $Y_{i}$. Let $\sigma$ be a global section of $\mathcal{O}^{\prime}$. Our claim will follow if we check that $\sigma$ can be regarded as a function on $X^{\prime}$ of weight $\chi$. To verify this we need to check that $\sigma_{i}(x)=\sigma_{j}\left(\varphi_{i j}^{-1} x\right)$, where $\sigma_{i}$ is the trivialization of $\sigma$. Here the subsets $\pi^{-1}\left(Y_{i j}\right) \subset \pi^{-1}\left(Y_{i}\right), \pi^{-1}\left(Y_{j}\right)$ are assumed to be identified as in $X$. But $\sigma_{j}\left(\varphi_{i j}^{-1} x\right)=\left\langle\chi, \varphi_{i j}\right\rangle^{-1} \sigma_{j}(x)=\sigma_{i}(x)$ (the factor $f_{i j}$ does not appear because of the choice of the identification of two different $\left.\pi^{-1}\left(Y_{i j}\right)\right)$.

Lemma 4.3. Let $c \in H^{2}(Y, \mathbb{C}) \hookrightarrow H^{2}\left(Y, \mathbb{C} \oplus \mathfrak{X}(T)^{*}\right) \cong H^{1}(Y, \mathcal{A} u t)$. Then $2 \pi i c=c_{0}-c_{0}^{\prime}$.

Proof. As in the proof of Lemma 2.7, we may assume that the actions $T$ : $X, T: X^{\prime}$ are free. There is an $\mathcal{O}(Y)$-valued 1-cochain $\left(f_{i j}\right)$ such that $\varphi_{i j}=$ $\exp \left(2 \pi i v\left(f_{i j}\right)\right)$. Let $\alpha_{i}$ be such as in the proof of Lemma 2.7. The 0 -cocycle 
in closed 1-forms constructed for $\omega^{\prime}$ as in the proof of Lemma 2.7 equals $\alpha_{j}-\varphi_{i j}^{*-1}\left(\alpha_{i}\right)=\left(\alpha_{j}-\alpha_{i}\right)+\alpha_{i}-\varphi_{i j}^{*-1}\left(\alpha_{i}\right)$. Let us compute $\alpha_{i}-\varphi_{i j}^{*-1} \alpha_{i}$. We may assume that $\pi^{-1}\left(Y_{i}\right)$ is an open neighborhood in $T^{*}(T)$. Let $\theta_{i}, \beta^{i}$ be such as in Example 2.2. Then $\alpha_{i}=\sum_{i=1}^{n} \beta^{i} d\left(\ln \theta_{i}\right)$. The map $\varphi_{i j}$ equals $\left(\theta_{i}, \beta^{i}\right) \mapsto\left(\theta_{i} \exp \left(2 \pi i \frac{\partial f_{i j}}{\partial \beta^{i}}\right), \beta^{i}\right)$. Thus

$$
\alpha_{i}-\varphi_{i j}^{*-1} \alpha_{i}=2 \pi i \sum_{k=1}^{n} \beta^{k} d\left(\frac{\partial f_{i j}}{\partial \beta^{k}}\right)=2 \pi i d\left(\sum_{k=1}^{n} \beta^{k} \frac{\partial f_{i j}}{\partial \beta^{k}}-f_{i j}\right) .
$$

So the 1-cocycle in $H^{1}\left(Y, \mathcal{O}_{Y} / \mathbb{C}\right)$ corresponding to $c_{0}^{\prime}-c_{0}$ is given by $2 \pi i\left(\mathcal{L} f_{i j}-f_{i j}\right)$, where $\mathcal{L}=\sum_{k=1}^{n} H_{i} \frac{\partial}{\partial H_{i}}$ (by abusing notation, $\frac{\partial}{\partial H_{i}}$ stands for the lifting of the corresponding vector field on $\mathfrak{t}^{*}$ to $Y$ ). Note that $\mathcal{L}\left(f_{i j}+f_{j k}+f_{k i}\right)=0$, for $f_{i j}+f_{j k}+f_{k i}=$ const. Therefore the classes of $c_{0}-c_{0}^{\prime}$ and $2 \pi i c$ in $H^{2}(Y, \mathbb{C})$ coincide.

Proof of Proposition 4.1. Let us prove that the action of $H^{1}(Y, \mathcal{A} u t)$ on $\mathbb{X}$ is transitive. Let $X, X^{\prime} \in \mathbb{X}$ and $\pi, \pi^{\prime}$ be the corresponding quotient maps. By Remark 2.9, there is an open covering $Y_{i}, i \in I$, of $Y$ such that there are Hamiltonian isomorphisms $\iota_{i}: \pi^{-1}\left(Y_{i}\right) \rightarrow \pi^{\prime-1}\left(Y_{i}\right), i \in I$, with $\pi^{\prime} \circ \iota_{i}=\pi$. So $\iota_{i} \circ \iota_{j}^{-1}$ is a 1-cocycle in $\mathcal{A} u t$ and $X^{\prime}=X_{c}$ for the corresponding cohomology class $c$. It follows that the action is transitive.

Choose $X \in \mathbb{X}$ and $\left(c_{i}^{\prime}\right)_{i=0}^{n} \in H^{2}\left(Y, \mathbb{C} \oplus \mathfrak{X}(T)^{*}\right)$. Let $\mathcal{Y}_{X}=\left(Y, \psi, \mathcal{D},\left(c_{i}\right)_{i=1}^{n}\right.$, $\left.c_{0}\right)$. By Lemma 4.2 , there is $c^{1} \in H^{2}\left(Y, \mathbb{C} \oplus \mathfrak{X}(T)^{*}\right)$ such that $\mathcal{Y}_{X_{c^{1}}}=$ $\left(Y, \psi, \mathcal{D},\left(c_{i}^{\prime}\right)_{i=1}^{n}, c_{0}^{\prime \prime}\right)$ for some $c_{0}^{\prime}$ and any two elements $c^{1}$ with this property differ by an element of $H^{2}(Y, \mathbb{C})$. Now, by Lemma 4.3 , there is a unique element $c^{2} \in H^{2}(Y, \mathbb{C})$ such that $\mathcal{Y}_{X_{c^{1} c^{2}}}=\left(Y, \psi, \mathcal{D},\left(c_{i}^{\prime}\right)_{i=1}^{n}, c_{0}^{\prime}\right)$. This completes the proof.

\section{Existence}

The goal of this section is to prove Theorem 1.2. Thanks to Proposition 4.1, it is enough to check that for any 5 -tuple $\mathcal{Y}$ of the form $\left(Y, \psi, \mathcal{D},\left(c_{i}\right)_{i=1}^{n}, c_{0}\right)$ with some $c_{i}, i=\overline{0, n}$, there is $X$ with $\mathcal{Y}_{X}=\mathcal{Y}$.

Choose an open covering $Y=\bigcup_{i} Y_{i}$ such that $Y_{i}$ is an open disk and there is an open saturated subset $X_{i}$ of some model Hamiltonian $T$-manifold such that $\mathcal{Y}_{X_{i}}=\left(Y_{i},\left.\psi\right|_{Y_{i}}, \mathcal{D}_{i}, 0,0\right)$, where $\mathcal{D}_{i}$ is the set of components of $D \cap$ $Y_{i}, D \in \mathcal{D}$. Let $\pi_{i}: X_{i} \rightarrow Y_{i}$ be the quotient map. Choose some isomorphisms $\iota_{i j}: \pi_{j}^{-1}\left(Y_{i j}\right) \rightarrow \pi_{i}^{-1}\left(Y_{i j}\right)$ of Hamiltonian manifolds such that $\pi_{i} \circ \iota_{i j}=\pi_{j}$. Such isomorphisms exist by Theorem 2.4. We may assume that $\iota_{i i}=i d, \iota_{j i}=$ $\iota_{i j}^{-1}$. Note, however, that, in general, $\iota_{i j} \iota_{j k} \iota_{k i} \neq i d$. So our task is to modify these isomorphisms for the cocycle condition to hold. 
There is $\zeta \in \mathfrak{X}(T) \otimes \mathbb{R}$ general for all $X_{i}$. Using Example 2.2, one easily gets that for any $i$ there is a subvariety $\widetilde{Y}_{i} \subset X_{i}^{\zeta}$ satisfying the following conditions:

(1) The restriction of $\pi_{i}$ to $\tilde{Y}_{i}$ is an isomorphism $\tilde{Y}_{i} \rightarrow Y_{i}$.

(2) $\tilde{Y}_{i}$ is a lagrangian submanifold of $X_{i}$.

Proposition 5.1. For any $i, j$ there is a unique element $\varphi_{i j} \in \mathcal{A} u t\left(Y_{i j}\right)$ such that

$$
\varphi_{i j}\left(\tilde{Y}_{i} \cap \pi_{i}^{-1}\left(Y_{i j}\right)\right)=\iota_{i j}\left(\tilde{Y}_{j} \cap \pi_{j}^{-1}\left(Y_{i j}\right)\right) .
$$

Proof. By Lemma 2.6, $\iota_{i j}\left(\pi_{j}^{-1}\left(Y_{i j}\right)^{\zeta}\right)=\pi_{i}^{-1}\left(Y_{i j}\right)^{\zeta}$. Since the group $\mathcal{A} u t\left(Y_{i j}\right)$ depends only on $Y_{i j}, \psi$ and does not depend on $\mathcal{D}$, we may assume that the actions $T: X_{i}, X_{j}$ are free. By (1), there is $\varphi_{i j} \in T_{Y}\left(Y_{i j}\right)$ satisfying (5.1). Note that $\varphi_{i j *} \xi_{*}=\xi_{*}, \varphi_{i j}^{*} H_{\xi}=H_{\xi}$ for any $\xi$. Therefore for any $x \in \pi_{i}^{-1}\left(Y_{i j}\right), \eta \in T_{x}\left(\pi_{i}^{-1}\left(Y_{i j}\right)\right)$ we have

$$
\omega_{\varphi_{i j}(x)}\left(\xi_{*}, \varphi_{i j *} \eta\right)=\partial_{\varphi_{i j *} \eta} H_{\xi}\left(\varphi_{i j} x\right)=\partial_{\eta} H_{\xi}(x)=\omega_{x}\left(\xi_{*}, \eta\right) .
$$

Since both $\widetilde{Y}_{i} \cap \pi_{i}^{-1}\left(Y_{i j}\right), \iota_{i j}\left(\widetilde{Y}_{j} \cap \pi_{j}^{-1}\left(Y_{i j}\right)\right)$ are lagrangian, we easily see that $\varphi_{i j}$ is a symplectomorphism. Thus $\varphi_{i j} \in \mathcal{A} u t\left(Y_{i j}\right)$.

Set $\widetilde{\iota}_{i j}:=\varphi_{i j}^{-1} \iota_{i j}$. By the construction of $\varphi_{i j}$,

$$
\widetilde{Y}_{i} \cap \pi_{i}^{-1}\left(Y_{i j}\right)=\widetilde{\iota}_{i j}\left(\widetilde{Y}_{j} \cap \pi_{j}^{-1}\left(Y_{i j}\right)\right) .
$$

This equation and (1) imply the cocycle condition $\widetilde{\iota}_{i j} \widetilde{\iota}_{j i} \widetilde{\iota}_{k i}=i d$.

Set $X:=\sqcup_{i} X_{i} / \sim$, where points $x_{i} \in X_{i}, x_{j} \in X_{j}$ are equivalent if $\pi_{i}\left(x_{i}\right)=\pi_{j}\left(x_{j}\right) \in Y_{i j}$ and $x_{i}=\widetilde{\iota}_{i j}\left(x_{j}\right)$. The manifold $X$ is Stein, see Proposition 2.10, it has the natural Hamiltonian structure and the action $T: X$ is MF. By construction, $\mathcal{Y}_{X}=\left(Y, \psi, \mathcal{D},\left(c_{i}\right)_{i=1}^{n}, c_{0}\right)$ for some $c_{i}$.

\section{An open problem}

Here we would like to state an open problem communicated to us by F. Knop. Until a further notice $T$ is a compact torus. Recall that any $\mathrm{MF}$ compact Hamiltonian $T$-manifold admits an invariant Kähler structure. This follows, for example, from the Delzant construction involving a Hamiltonian reduction.

For a moment, let $X$ be an arbitrary smooth manifold. Recall that a hyperkähler structure on $X$ is a quadruple $(q, I, J, K)$, where $q$ is a Riemannian metric, and $I, J, K$ are complex structures satisfying the quaternionic relations $I J=K, J K=I, K I=J$ and such that the three 2-forms defined by $\omega_{A}(u, v)=q(A u, v), A=I, J, K$, are symplectic. The basic example here is $X=\mathbb{R}^{4 n}$ considered as an $n$-dimensional quaternionic vector space $\mathbb{H}^{n}$. Note that $\omega_{J}+i \omega_{K}$ is a holomorphic symplectic form with respect to the complex structure $I$. 
Now let $X$ be a hyperkähler manifold equipped with an action of $T$ preserving the hyperkähler structure. This action is called hyperhamiltonian if it is Hamiltonian for all symplectic forms $\omega_{A}, A=I, J, K$. Let $\mu_{I}, \mu_{J}, \mu_{K}$ be the corresponding moment maps. Then one easily checks that $\mu_{J}+i \mu_{K}$ is a holomorphic map with respect to the complex structure $I$. If one can lift the action $T: X$ to a holomorphic action $T_{\mathbb{C}}: X$, where $T_{\mathbb{C}}$ denotes the complexification of $T$, then $X$ becomes a Hamiltonian $T_{\mathbb{C}-\text { manifold. }}$

We say that a hyperhamiltonian action $T: X$ is $\mathrm{MF}$ if $\operatorname{dim} X=4(\operatorname{dim} T-$ $\operatorname{dim} T_{0}$ ), where $T_{0}$ denotes the inefficiency kernel for the action $T: X$.

Actually, there is a special class of MF hyperhamiltonian actions studied extensively in the last 10 years, so-called hypertoric manifolds (or toric hyperkähler manifolds), see, for example, $[\mathbf{B D}],[\mathbf{H S}],[\mathbf{K}],[\mathbf{P}]$. Let us give their definition.

There is a reduction procedure for hyperhamiltonian manifolds introduced in a more general setup in [HKLR]. Choose a connected subgroup $T_{0} \subset T$. For $\alpha \in\left(\mathfrak{t}_{0}^{*}\right)^{\oplus 3}, \alpha=\left(\alpha_{1}, \alpha_{2}, \alpha_{3}\right)$, we put $X / / /_{\alpha} T:=\left(\mu_{0}^{-1}(\alpha)\right) / T_{0}$, where $\mu=\left(p \circ \mu_{I}, p \circ \mu_{J}, p \circ \mu_{K}\right), p: \mathfrak{t}^{*} \rightarrow \mathfrak{t}_{0}^{*}$ is the natural projection. If $T_{0}$ acts freely on $\mu^{-1}(\alpha)$, then $X / / /{ }_{\alpha} T_{0}$ is a genuine manifold of dimension $\operatorname{dim} X-4 \operatorname{dim} T_{0}$ possessing a natural hyperkähler structure. Moreover, $X$ is a hyperhamiltonian $T / T_{0}$-manifold. By a hypertoric manifold one means a reduction of $\mathbb{H}^{n}$ by a subtorus in a maximal torus of $\operatorname{Sp}(n)$.

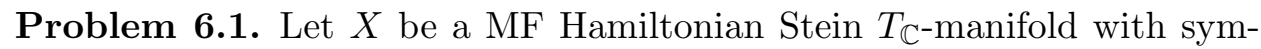
plectic form $\omega$ and moment map $\mu$. Does there exist a hyperkähler structure $(q, I, J, K)$ on $X$ such that

(1) $I$ coincides with the initial complex structure on $X$.

(2) $\omega_{J}=\operatorname{Re} \omega, \omega_{K}=\operatorname{Im} \omega$.

(3) The action $T: X$ is hyperhamiltonian with $\mu_{J}=\operatorname{Re} \mu, \mu_{K}=\operatorname{Im} \mu$.

\section{References}

[A] M. Atyah, Convexity and commuting Hamiltonians, Bull. London. Math. Soc. 14 (1982), 1-15.

[BD] R. Bielawsky and A. Dancer, The geometry and topology of toric hyperkähler manifolds, Comm. Anal. Geom. 8 (2000), 727-760.

[D] T. Delzant, Hamiltoniens périodiques et images convexes de l'application moment, Bul. Soc. Math. France 116 (1988), 315-339.

[GS] V. Guillemin and Sh. Sternberg, Convexity properties of the moment mappings, Invent. Math. 67 (1982), 491-513.

[HKLR] N. Hitchin, A. Karlhede, U. Lindström and M. Roček, Hyper-Kähler metrics and supersymmetry, Comm. Math. Phys. 108 (1997), 535-589.

[HS] T. Hausel and B. Sturmfels, Toric hyperkähler varieties, Doc. Math. 7 (2002), 495-534.

[HMP] P. Heinzner, L. Migliorini and M. Polito, Semistable quotients, Ann. Sc. Norm. Super. Pisa Cl. Sci. (4), 26 (1998), 233-248. 
[K] H. Konno, Cohomology rings of toric hyperkähler varieties, Int. J. Math. 11 (2000), 1011-1026.

[L] I.V. Losev, Symplectic slices for reductive groups, Mat. Sbornik 197 (2006), N2, 75-86 (in Russian), English translation in: Sbornik Math. 197 (2006), N2, 213224 .

[O] A.L. Onishchik, Methods of sheaf theory and Stein spaces, Itogi nauki i tekhniki, Sovr. Prob. Mat., Fund. Napr, Vol. 10, 5-83. Moscow, VINITI, 1986 (in Russian).

[P] N. Proudfoot, Hyperkähler analogues of Kähler quotients, Preprint (2004), arXiv:math.AG/0405233.

[S] D.M. Snow, Reductive group actions on Stein spaces, Math. Ann. 259 (1982), 79-97.

[W] C. Woodward, The classification of transversal multiplicity-free Hamiltonian actions, Ann. Global. Anal. Geom. 14 (1996), 3-42.

Department of Mathematics

Massachusetts Institute of Technology

CAmbridge, MA 02139, USA

E-mail address: ivanlosev@yandex.ru; ivanlosev@math.mit.edu

Received 7/17/2007, accepted 9/18/2008

Part of the work on this problem was done during author's stay in Rutgers University, New Brunswick, in the beginning of 2007. I would like to thank this institution and especially Professor F. Knop for hospitality. I also express my gratitude to F. Knop for stimulating discussions. Finally, I would like to thank J. Martens and the referee for useful comments on earlier versions of this article. 